\title{
Plasmon resonance induced photoconductivity in the yttria stabilized zirconia films with embedded Au nanoclusters
}

(C) D.O. Filatov, I.N. Antonov, D.Yu. Sinutkin, D.A. Liskin, A.P. Gorshkov, O.N. Gorshkov,

V.E. Kotomina, M.E. Shenina, S.V. Tikhov, I.S. Korotaeva

Lobachevskii State University of Nizhny Novgorod,

603950 Nizhny Novgorod, Russia

E-mail: dmitry_filatov@inbox.ru

We report on the investigation of the photoconductivity (PC) in the yttria stabilized zirconia (YSZ) films with embedded Au nanoclusters (NCs). A peak in the PC spectrum corresponding to the plasmon optical absorption resonance in the Au NCs was observed. The temperature dependence of PC near $300 \mathrm{~K}$ obeyed Mott law. In this case, the PC was attributed to the heating of the YSZ matrix due to the plasmon optical absorption in the Au NCs (bolometric effect). Near $77 \mathrm{~K}$, the PC did not depend on temperature and was attributed to plasmon-assisted electron transport between the NCs via the vacancy $\alpha$-band in YSZ.

\section{Acknowledgements}

This work has been supported by Ministry of Education and Science of Russian Federation (Project 16.7864.2017). The PC measurements have been performed using the shared research facilities of Research and Educational Center for Physics of Solid State Nanostructures at Lobachevskii State University of Nizhny Novgorod. 